\title{
Avaliação Cardiovascular de Pacientes Diabéticos em Ambulatório Universitário
}

\begin{abstract}
RESUMO
Introdução: Baseado no conhecimento atual sobre o diabetes tipo 2 (DM), considerado como um estado de doença cardiovascular, várias diretrizes surgem no intuito de sugerir uma avaliação cardiovascular rotineira nesses indivíduos, na tentativa de se detectar precocemente a doença arterial coronariana (DAC). Neste trabalho, objetivamos descrever qual avaliação cardiovascular basal fora realizada em um período de seguimento dos mesmos, dentro de um centro universitário especializado no tratamento de diabetes, adequado para a realidade brasileira. Sujeitos e Métodos: Anotamos dados clínicos do prontuário de 121 pacientes portadores de DM tipo 2 atendidos no Centro de Diabetes da Universidade Federal de São Paulo e confrontamos com o tipo de avaliação cardiovascular a que os mesmos haviam sido submetidos no ano que precedia a consulta. Resultados: Observamos que na população estudada havia uma alta prevalência de outros fatores de risco cardiovasculares associados. Quanto às avaliações cardiovasculares realizadas no período, cerca de $36 \%$ dos pacientes não realizaram nenhum teste cardiológico, $17 \%$ foram submetidos ao eletrocardiograma de repouso e $27 \%$ dos pacientes foram submetidos ao teste ergométrico. Exames como ecocardiograma em repouso ou sob stress farmacológico, cintilografia de perfusão do miocárdio e cinecoronariografia foram realizados em menor proporção. Conclusão: Nossos dados mostraram a variabilidade e limitações na forma de abordagem diagnóstica de DAC de pacientes acompanhados em ambiente universitário e nos apontam para a necessidade de construir diretrizes mais bem definidas e dirigidas para as peculiaridades da população e sistema de saúde brasileiros. (Arq Bras Endocrinol Metab 2007;51/2:281-284)
\end{abstract}

Descritores: Diabetes mellitus; Doença cardiovascular; Doença arterial coronariana

\begin{abstract}
Cardiovascular Assessment of Diabetic Patients.

Introduction: Type 2 diabetes is a cardiovascular disease. The morbidity and mortality among these patients are primarily due to cardiovascular diseases. There are many guidelines regarding clinical evaluation of cardiovascular disease in those patients. Implementation of these guidelines has been an argued subject. Our objective in this paper is to describe what basal cardiovascular evaluation has been carried out at a specialized university Diabetes Center. Subjects and Methods: Data were collected from February to October 2006 of 121 type 2 diabetes individuals who were enrolled at the Diabetes Center of Federal University of São Paulo. We analyzed the type of cardiovascular disease evaluation that they had been submitted in the year that preceded the consultation. Results: We have observed a high prevalence of several other cardiovascular risk factors in this population. The cardiovascular evaluations during this period has shown $36 \%$ of the patients had not been submitted to any cardiovascular test, $17 \%$ had been submitted to resting electrocardiogram and $27 \%$ of the patients had been submitted to exercise test. Rest echocardiogram, pharmacologic stress echocardiogram, myocardial perfusion scintigraphy, and coronary angiography have been carried out in a much lesser ratio. Conclusion: Our data has shown the variability and limitations on boarding diagnosing of DAC in university environment patients and point us the necessity of constructing defined and directed directives for the peculiarities of the Brazilian population and health system. (Arq Bras Endocrinol Metab 2007;51/2:281-284)
\end{abstract}

Keywords: Diabetes mellitus; Cardiovascular disease; Coronary disease artigo original

\author{
Marcelo A. Alvarenga \\ Carolina S.V. OliveIRA \\ ANTONNIO R. CHACRA \\ ANDRÉ F. REIS
}

Centro de Diabetes, Disciplina

de Endocrinologia,

Universidade Federal de

São Paulo - UNIFESP/Escola

Paulista de Medicina - EPM,

São Paulo, SP.

Recebido em 04/01/07

Aceito em 09/01/07 
$\mathrm{O}$ DiABETES MELlitus (DM) é um dos principais fatores de risco para doença cardiovascular, sendo que a doença arterial coronariana (DAC) representa cerca de $75 \%$ das causas de morte nos portadores de DM. Ainda, a isquemia silenciosa do miocárdio é mais freqüente em diabéticos, sendo sempre um desafio diagnóstico na prática clínica (1-4). Recentemente, surgiu o conceito de que o DM seria um estado de equivalência de doença coronariana, o que estimula intensos debates no sentido da abordagem destes pacientes com o objetivo de redução da DAC nestes indivíduos. Ainda, nos últimos 5 anos, fortes evidências surgiram no sentido da ampliação do uso de medicamentos cardioprotetores no DM, tais como redutores do colesterol, aspirina, betabloqueadores e inibidores da enzima de conversão a angiotensina (5). Da mesma forma, ocorreu um grande avanço no campo da detecção da DAC com o emprego de novos testes diagnósticos e graduação da DAC, menos invasivos que a cineangiocoronariografia, considerada como padrão ouro (6).

Acredita-se que com as novas formas de abordagem e, sobretudo, com o estabelecimento de rotinas e protocolos mais bem definidos seja possível obter diagnósticos mais precoces, o que pode resultar em importante impacto tanto na gravidade quanto na mortalidade por DAC nos pacientes com diabetes (5). Enquanto vários consensos internacionais procuram definir ou orientar os clínicos sobre quais pacientes com diabetes deveriam ser testados para DAC e, ainda, qual o teste cardiológico mais indicado, não existe pleno consenso sobre esses aspectos (7-11). Para construção de protocolos de diagnóstico mais eficazes para uma determinada população é necessário dispor de um perfil mais detalhado acerca da mesma, além de outros pontos importantes. Desta forma, torna-se necessária a descrição de características específicas de cada população, onde efeitos genéticos têm papel marcante na apresentação, prevalência de gravidade da DAC (12), prevalência de fatores de risco associados, condições sócio-econômicas locais e regionais específicas, inerentes a cada sistema de saúde, e a definição de quais testes diagnósticos estão disponíveis para aquela situação.

Neste presente estudo, objetivamos descrever a forma com que são abordados os pacientes com DM acompanhados em um ambulatório de referência no Brasil, dentro de uma universidade. Esta análise poderá ser útil na composição dos elementos que permitam, no futuro, a elaboração de condutas de diagnóstico de DAC com elementos próprios da realidade brasileira. Ainda, os dados nos trazem a possibilidade de uma otimização no processo de rotina diagnóstica destes casos.

\section{SUJEITOS E MÉTODOS}

No período de fevereiro a outubro de 2006, durante as consultas médicas realizadas no Ambulatório do Centro de Diabetes da Escola Paulista de Medicina - UNIFESP, anotamos em dados do prontuário, de forma retrospectiva, o perfil de 121 pacientes portadores de DM tipo 2 (definida a princípio por uma somatória de dados que sugeriam como tal: idade de início dos sintomas após 25 anos, sobrepeso ou obesidade associados e a não-necessidade do uso de insulina, ao menos nos primeiros anos de diagnóstico). Anotamos também a qual avaliação cardiovascular os mesmos haviam sido submetidos durante o ano que precedia a consulta, fossem estes pacientes assintomáticos do ponto de vista cardiovascular (exame de rotina) ou sintomáticos (busca ativa de um diagnóstico). Oito parâmetros, considerados como fatores de risco para DCV, foram anotados dos prontuários dos pacientes: 10 anos)

1) tempo de diagnóstico do diabetes (> 10 anos ou $<$

2) presença de complicações crônicas microvasculares: retinopatia (ao exame de fundo de olho) e/ou nefropatia (albuminúria $>20 \mu \mathrm{g} / \mathrm{min}$ em amostra de urina de 12 horas, presente em duas medidas distintas) e/ou neuropatia periférica (queixas clínicas e/ou alteração ao exame físico da sensibilidade tátil - monofilamento $10 \mathrm{~g}$; vibratória - diapasão $128 \mathrm{~Hz}$; dolorosa - palito; reflexos tendinosos aquileu e patelar) e/ou neuropatia autonômica (presente ou provável, definida por hipotensão postural $>20 \mathrm{mmHg}$ na PAS ou $10 \mathrm{mmHg}$ na PAD após o $3^{\circ}$ minuto, taquicardia de repouso - FC > 100 bpm, disfunção erétil);

3) presença de complicações crônicas macrovasculares: DAC estabelecida (IAM prévio, cirurgia de revascularização do miocárdio ou stent coronariano prévio) ou suspeita (angina pectoris - estável ou instável) e/ou acidente vascular cerebral prévio e/ou doença arterial periférica obstrutiva crônica sintomática (claudicação intermitente, dor em repouso ou antecedente de revascularização arterial de membros inferiores);

4) HAS (PA $\geq 130 \times 80 \mathrm{mmHg}$ em pelo menos duas ocasiões distintas, com intervalo de 2 semanas no mínimo entre as medidas ou o uso de medicações anti-hipertensivas);

5 ) dislipidemia (colesterol total $\geq 200 \mathrm{mg} / \mathrm{dL}, \mathrm{LDL}$ $\geq 100 \mathrm{mg} / \mathrm{dL}, \mathrm{HDL} \leq 45 \mathrm{mg} / \mathrm{dL}$ nos homens ou $\leq 55$ $\mathrm{mg} / \mathrm{dL}$ nas mulheres e $\mathrm{TG} \geq 150 \mathrm{mg} / \mathrm{dL}$ ou uso de medicações hipolipemiantes);

6) obesidade (IMC $\left.\geq 30 \mathrm{~kg} / \mathrm{m}^{2}\right)$;

7) tabagismo atual;

8 ) antecedente familiar de doença aterosclerótica prematura (parente de $1^{\circ}$ grau do sexo masculino $\leq 55$ anos ou feminino $<65$ anos).

Em uma outra análise, agrupamos os pacientes como um todo em dois outros grupos segundo uma graduação de risco clínico para doença cardiovascular em que se enquadravam. Os grupos foram definidos da seguinte maneira: Baixo Risco - presença de 1 ou nenhum dos parâmetros citados acima, associados ao DM; Alto Risco - 
presença de 2 ou mais dos parâmetros citados acima, associados ao DM (9-10).

Quanto às avaliações cardiovasculares realizadas no ano que precedia a consulta, observamos que diversas situações ocorriam em relação ao tipo de avaliação para a busca da presença de doença cardiovascular:

- nenhum teste ou exame realizados (apenas a consulta clínica);

- ECG basal;

- teste ergométrico (esteira ou bicicleta);

- ECO transtorácico em repouso;

- ECO transtorácico sob stress farmacológico (dipiridamol ou dobutamina);

- cintilografia do miocárdio / MIBI (após stress farmacológico ou após teste ergométrico);

- cineangiocoronariografia (cateterismo cardíaco).

\section{RESULTADOS}

A população estudada apresentava idade entre 27 e 79 anos (57,6 anos $\pm 13,8$; média \pm DP) sendo que $45 \%$ eram do sexo masculino. Na população como um todo, observamos uma alta prevalência de outros fatores de risco (tabela 1) para doença cardiovascular, o que os classificava como alto risco cardiovascular na maioria dos casos $(92 \%)$.

Quanto à avaliação cardiovascular realizada, observamos que 59 pacientes ( $49 \%$ do total) foram submetidos a algum tipo de avaliação cardiovascular não-invasiva. No entanto, dos exames realizados, com comprovada acurácia diagnóstica, houve evidente predomínio da realização do teste de esforço (ergométrico), ou seja, $27,2 \%$ dos casos (33 pacientes). Sete pacientes foram avaliados com cineangiocoronariografia (cateterismo cardíaco), exame padrão ouro para a avaliação $(7,10)$. A cintilografia e o ecocardiograma sob stress farmacológico, considerados como testes de escolha para uma boa parcela dos pacientes

Tabela 1. Estatística geral dos fatores de risco avaliados em 121 pacientes com DM tipo 2.

\begin{tabular}{lcc}
\hline & $\mathbf{n}$ & \% Total \\
\hline 10 anos DM & 70 & $57,8 \%$ \\
\hline Complicações microvasculares & 53 & $43,8 \%$ \\
\hline Complicações macrovasculares & 29 & $23,9 \%$ \\
\hline HAS & 98 & $80,9 \%$ \\
\hline Dislipidemia & 85 & $70,2 \%$ \\
\hline Obesidade & 28 & $23,1 \%$ \\
\hline Tabagismo & 16 & $13,2 \%$ \\
\hline Antecedente familiar DAC & 20 & $16,5 \%$ \\
\hline Total & 121 & $100 \%$ \\
\hline
\end{tabular}

Tabela 2. Avaliação cardiovascular realizada e sua porcentagem na população estudada.

\begin{tabular}{lcc}
\hline & $\mathbf{n}$ & $\%$ \\
Nenhuma avaliação & 42 & $34,7 \%$ \\
ECG repouso & 20 & $16,5 \%$ \\
Teste ergométrico & 33 & $27,2 \%$ \\
ECO repouso & 08 & $6,6 \%$ \\
ECO stress & 04 & $3,3 \%$ \\
Cintilografia de perfusão & 07 & $5,8 \%$ \\
Cineangiocoronariografia & 07 & $5,8 \%$ \\
Total & 121 & $100 \%$ \\
\hline
\end{tabular}

avaliados (10), representaram aproximadamente 9\% das avaliações observadas. Por outro lado, cerca de $35 \%$ dos casos não realizaram teste algum no último ano (42 pacientes) e 16\% realizaram apenas o eletrocardiograma em repouso (20 pacientes).

\section{DISCUSSÃO}

Nossos dados demonstram uma grande variabilidade na forma de abordagem de doença cardiovascular em pacientes com DM tipo 2 acompanhados em ambulatório de diabetes dentro de um ambiente universitário. Se, por um lado, cerca de $48 \%$ dos indivíduos foram submetidos a algum tipo de exame não-invasivo com boa acurácia diagnóstica (11), como o teste ergométrico, ecocardiograma com ou sem stress farmacológico e a cintilografia do miocárdio, exigindo uma estrutura mais especializada (típica de centros universitários), cerca de $50 \%$ dos indivíduos não receberam nenhuma avaliação adicional após a consulta clínica ou realizaram apenas o eletrocardiograma em repouso. Aproximadamente 35\% dos pacientes não dispõem de qualquer tipo de avaliação cardiovascular (apenas exame clínico).

É importante ressaltar que, com muita freqüência, os pacientes recebiam a orientação de realizar algum teste, especialmente o ergométrico. Porém, com o excesso de pacientes que o sistema converge e também pelo nível de complexidade (nível terciário) dos mesmos, parte deles apresentou dificuldades adicionais com relação ao processo de agendamento de consultas e dos exames. De fato, durante os atendimentos vários pacientes relataram dificuldades em agendar os exames solicitados pela equipe, sendo, portanto, enquadrados em nossa análise no grupo de pacientes sem avaliação cardiovascular recente. Esta dificuldade em agendar o exame poderia gerar atraso na avaliação cardiovascular desejada e conseqüentemente na terapêutica mais adequada para a situação. 
Nota-se que, de acordo com a literatura, a maior parte dos pacientes portadores de DM tipo 2 apresenta outros fatores de risco associados, caracterizando-os como um grupo de alto risco para doença cardiovascular. Apesar de o rastreamento de DAC indiscriminado não ser indicado, como valor clínico ainda discutível, a população estudada é bastante representativa de pacientes portadores de DM tipo 2 com alto risco cardiovascular, merecendo algum tipo de avaliação cardiovascular $(8,9,11)$. Segundo algumas entidades científicas de diabetes como a ADA (americana) e a ALFEDIAM (francesa), a maior parte ( $95 \%)$ dos pacientes estudados mereceria algum tipo de avaliação cardiológica dentre teste ergométrico, eco stress ou cintilografia de perfusão do miocárdio $(8,9,11)$.

Ressaltamos que a nossa casuística é limitada, porém a consideramos representativa da realidade da maior parte dos centros ambulatoriais universitários especializados no atendimento em diabetes. Diante das dificuldades citadas, dentre outras, optamos por não descrever os achados dos exames solicitados, uma vez que não tivemos acesso a exames padrão ouro para a avaliação cardiovascular dos pacientes, o que limitaria a nossa análise. Um estudo com mulheres brasileiras diabéticas e assintomáticas demonstrou uma prevalência de aproximadamente $30 \%$ de lesões graves coronarianas (> 50\% de obstrução) ao cateterismo, corroborando mais uma vez para a idéia de se fazer uma busca ativa de DAC em portadores de DM (6).

Neste estudo, descrevemos um retrato da forma de abordagem de DCV dos pacientes acompanhados no centro de diabetes de um ambiente universitário. Notamos que, se por um lado, uma boa parcela dos pacientes dispõe de testes não-invasivos com complexidades variadas, uma outra grande parcela apresenta dificuldades de acesso à realização dos testes por provável excesso na demanda do sistema de saúde. Uma abordagem multidisciplinar com participação ativa de endocrinologistas, diabetologistas e cardiologistas poderá otimizar o encaminhamento desses casos, tornando as indicações mais eficazes com um menor tempo de espera.

A elaboração de um protocolo de encaminhamento das solicitações dos testes cardiológicos poderá identificar de forma mais eficaz e rápida os indivíduos com DCV, propiciando terapêutica precoce e minimizando a morbidade e mortalidade deste pacientes.

\section{REFERÊNCIAS}

1. The effect of intensive treatment of diabetes on the development and progression of long-term complications in insulindependent diabetes mellitus. The Diabetes Control and Complications Trial Research Group. N Engl J Med 1993; 329(14):977-86.

2. Effect of intensive blood-glucose control with metformin on complications in overweight patients with type 2 diabetes (UKPDS 34). UK Prospective Diabetes Study (UKPDS) Group. Lancet 1998;352(9131):854-65.

3. Kannel WB, McGee DL. Diabetes and cardiovascular disease. The Framingham study. JAMA 1979;241:2035-8.

4. Wackers FJ, Young LH, Inzucchi SE, Chyun DA, Davey JA, Barrett EJ, et al. Detection of ischemia in asymptomatic diabetics investigators: detection of silent myocardial ischemia in asymptomatic diabetic subjects: the DIAD study. Diabetes Care 2004;27:1954-61.

5. Booth GL, Kapral MK, Fung K, Tu JV. Relation between age and cardiovascular disease in men and women with diabetes compared with non-diabetic people: a population based retrospective cohort study. Lancet 2006;368:29-36.

6. Smanio P. Doença cardiovascular em mulheres diabéticas assintomáticas. Arq Bras Endocrinol Metab 2007;51(2): in press.

7. American Diabetes Association: Consensus development conference on the diagnosis of coronary heart disease in people with diabetes: 10-11 February 1998, Miami, Florida. Diabetes Care 1998;21:1551-9.

8. Young LH, Jose P, Chvun D. Diagnosis of CAD in patients with diabetes: who to evaluate? Curr Diab Rep 2003;3:1927.

9. Standards of Medical Care in Diabetes - 2006, American Diabetes Association. Diabetes Care 2006;29:S4-42.

10. Puel J, Valensi P, Vanzetto G, Lassmann-Vague V, Monin JL, Moulin P, et al.; ALFEDIAM-SFC. Identification of myocardial ischemia in the diabetic patient. Joint ALFEDIAM and SFC recommendations. Diabetes Metab 2004;30:3S3-18.

11. Le Feuvre $C$, Barthélémy $O$, Dubois-Laforgue D, Maunoury Ch, Mogenet A, Baubion N, et al. Stress myocardial scintigraphy and dobutamine echocardiography in the detection of coronary disease in asymptomatic patients with type 2 diabetes. Diabetes Metab 2005;31:135-42.

12. Fumeron F, Reis AF, Velho G. Genetics of macrovascular complications in diabetes. Curr Diab Rep 2006;6:162-8.

Endereço para correspondência:

André F. Reis

Rua Gal. Waldomiro de Lima 508

04344-070 São Paulo, SP

E-mail: andré.reis@fleury.com.br 